\title{
VERSITA
}

Journal of Official Statistics, Vol. 29, No. 1, 2013, pp. 49-71, DOI: 10.2478/jos-2013-0004

\section{Redesign of Statistics Production within an Architectural Framework: The Dutch Experience}

\author{
Peter Struijs ${ }^{1}$, Astrea Camstra ${ }^{1}$, Robbert Renssen ${ }^{1}$, and Barteld Braaksma ${ }^{1}$
}

\begin{abstract}
In response to a changing environment, Statistics Netherlands has embarked on a large-scale redesign of the way statistics are produced. The aim is to increase the capability to respond to changing information demand, to lower the response burden for surveys, especially for businesses, and to improve efficiency, while preserving the overall quality level. The redesign is carried out within the framework of a so-called enterprise architecture, which gives overall guidance when structuring the processes of the organisation, including statistical methods and IT tools used. The article describes the redesign approach and explains the main features of the architecture. The emphasis is on experiences that may be relevant to other national statistical institutes operating in a similar environment.
\end{abstract}

Key words: Process design; enterprise architecture; IT expenditure; standardisation; GSBPM.

\section{Introduction}

The context in which official statistics are produced by national statistical institutes (NSIs) has profoundly changed in the last decades (Van der Veen 2007). Many NSIs are experiencing budgetary tightening and political pressure to reduce the administrative burden of survey participation, especially in the business sector (Ypma and Zeelenberg 2007). At the same time, NSIs are facing an ever-growing demand for statistical information. This relates to new and changing user needs for relevant statistics, for example about the financial downturn or immigration, as well as to the timeliness and amount of detail with which statistics are to be released. In the European Union (EU), the number of regulations requiring EU member states to produce statistics has multiplied in the last two decades, for instance in the area of short-term statistics and annual business statistics.

In addition, technological advances have resulted in more possibilities for survey and automated data collection, and administrative registers have increasingly become available for statistical purposes. The amount of data available for use as input for statistics has grown significantly and requires integrating different data sources in an intelligent way.

1 Statistics Netherlands, Postbus 24500, 2490 HA, Den Haag, The Netherlands. Website www.cbs.nl, for all authors. Emails: psjs@cbs.nl, acma@cbs.nl, rrnn@cbs.nl, and bbka@cbs.nl, respectively.

Acknowledgments: The authors thank Geert Bruinooge, Dirkjan Beukenhorst, Frank Hofman and Kees Zeelenberg, Statistics Netherlands, for their contribution to the discussion and their comments on drafts of the article. Comments by the guest editors of JOS were especially valuable. The views expressed in this article are those of the authors and do not necessarily reflect the position of Statistics Netherlands. 
To meet the challenges of a transforming environment, NSIs will have to adapt the way in which they produce statistics. This is widely acknowledged (Bae 2010; Braaksma et al. 2013; Doherty 2010; Falorsi et al. 2013; Galvin and Rhodes 2010; Lorenc et al. 2011; Studman 2010; Swiss Federal Statistical Office 2007). At Statistics Netherlands (SN), the following key strategic goals have been formulated to guide this transformation:

1. It must be possible to quickly and flexibly respond to changing information demand.

2. The existing high quality standards must be maintained and coherence improved.

3. The reporting burden should be minimal, especially for businesses.

4. The statistical processes must be cost efficient.

Although other NSIs face more or less the same challenges, they may choose to concentrate on somewhat different strategic goals. The strategic aims themselves are not the focus of this article, but rather the way in which they can be achieved. Translating the strategy into the necessary organisational changes is where an enterprise architecture can be of value.

Briefly, an enterprise architecture can be seen as a general blueprint of how to create an organisation's processes and IT infrastructure (Lankhorst 2009). It can serve as a guideline for the redesign projects that are carried out to bring about the changes as well as for the management of change. The Generic Statistical Business Process Model (GSBPM) is an international reference for describing statistical production processes used by NSIs at a high level (Vale 2009). However, this model only covers part of an enterprise architecture as it mainly focuses on statistical processes.

This article illustrates how the enterprise architecture developed and used by SN contributes to realising its strategic goals. SN began developing the architecture in 2004 . The purpose of the article is not to give a historic account, but to identify factors, pitfalls and practices that may be relevant to other NSIs operating in a similar environment and facing similar challenges. Obviously, the enterprise architecture has been developed given a specific context. $\mathrm{SN}$ is a centrally organised institute that is responsible for providing 95\% of official statistics in the Netherlands. This situation is quite different from that in other countries where there are several producers of official statistics like ministries and regional agencies. Institutional independence is guaranteed by law. European statistical requirements have a serious effect on the work programme of $\mathrm{SN}$ : more or less $70 \%$ is determined by European regulations, which are legally binding for the EU member states. Nevertheless, most key elements of the enterprise architecture - and the architectural approach itself - are independent of the local context.

We start in Section 2 with arguing for an integral approach to producing statistics, moving away from the traditional approach of independent, parallel production lines (sometimes called 'stovepipes' or 'silos'). The integral approach is incorporated in the enterprise architecture of SN, which is described in Section 3, together with an example of its application in economic statistics. The section illustrates how the architecture makes the strategic goals operational. In particular, it shows how the architecture can help to increase the responsiveness to new information needs and reduce the response burden. Having an enterprise architecture alone is not sufficient to realise the desired transformations. An important factor that also contributes to the achievement of the organisational goals is the standardisation of statistical methods and IT applications 
(Braaksma 2009). This is the subject of Section 4. The availability of such standards enhances the transparency and flexibility of the design of the statistical production process, thus increasing the potential for reuse and hence reducing costs. Greater transparency in designing production processes is also expected to result in a better grip on the quality of the statistical data produced. And it is important to embed the enterprise architecture in the organisation in order to successfully implement the ensuing redesign programme. Section 5 describes two organisational arrangements that have been made: a board for architectural issues and a board for portfolio decisions. Decisions on the enterprise architecture, on standards and on the implementation of the redesign programme are to be based on cost-benefit considerations. Section 6 is devoted to this side of applying an integral approach to statistics. Section 7 summarises our experiences and highlights some issues to be dealt with in the future, including international cooperation.

\section{The Need for an Integral Approach}

Traditionally, statistics of most NSIs are typically produced in more or less separate and autonomous production lines, each having their own methodology and IT systems. In a nutshell, the conventional way of producing statistics consists of surveying a sample or census of persons, households, businesses or institutions with a questionnaire, processing the data using appropriate methodology, compiling tables and explanations, and publishing results on the underlying population. This way of producing statistical information places much emphasis on the development of efficient methodology for data collection, data editing and analysis. Over many years, the accepted statistical methods and techniques have become increasingly sophisticated.

The changes in the environment in which they operate have prompted NSIs to re-examine their methodological research programmes (HLG-BAS 2012). In the field of data collection, the research is now more focussed on the use of administrative registers and mixed-mode survey strategies, preferably using modes with lower per-unit cost (e.g., Internet). The new ways of data collection in turn call for advanced estimation methods for combining data from various sources, or for small areas (domains) where by design few data points are collected. The ambition to publish statistics more rapidly or work more efficiently has likewise driven improvements in macro-level editing and imputation techniques.

However, new and improved methods alone are not sufficient to completely attain the four strategic goals SN has set for itself, as they leave the traditional approach to producing statistics intact. There are two main reasons why retaining autonomous production lines may impede reaching the goals.

First, as independent production lines for statistical products typically have their own design, customised methodology and IT solutions, they are expensive to develop and maintain. Adopting a more integral approach to designing statistical production processes creates opportunities for economies of scale in process design, statistical methods, IT applications, development, training, and so on.

At SN, the costs of IT maintenance are particularly relevant. A decade ago, the statistical departments often had their own IT solutions, which were in many cases maintained locally. In fact, SN still has to deal with a large legacy of IT systems and their 
increasing maintenance costs. The sustainability of such solutions was eventually questioned. Incidentally, many large organisations face or have faced these problems, which can be seen as a natural corollary to the exponential growth of IT in the last few decades (Engelbertink and Vogt 2010).

Second, although the designs of autonomous production lines may be optimised from a local point of view with respect to certain dimensions such as output quality and processing costs, there is no incentive to optimise these designs from a corporate point of view. Especially issues dealing with the coherence of statistics, but also the reduction of the reporting burden and the reuse of IT solutions should be tackled companywide. Moreover, autonomous production lines make it difficult to combine and reuse already available statistical data, which is necessary for a quick response to new information needs.

Full optimisation is the Holy Grail of so-called total survey design. However, a more integrated approach to statistics production requires optimising the set of production lines as a whole, that is, across the whole organisation. Reduction of response burden, for instance, is possible when the same source data can be used in more than one production line and, conversely, a specific set of statistical tables may be based on a number of sources. This leads to an interconnected set of production lines, that is, a network, rather than the traditional set of autonomous lines. In such a situation, one should ideally aim at total network design rather than total survey design. The network is optimised for the same dimensions as is the case when applying total survey design, but the optimisation is global rather than local. This does not imply that tailor-made production lines have to be totally banned, only that they have to be subjected to an organisation-wide assessment.

The need to design production lines collectively from a company-wide perspective calls for an enterprise architecture, which serves to a link pin between the strategic goals at the level of the organisation with the individual production lines used for producing the statistics.

\section{An Outline of the Enterprise Architecture for Statistics Production}

When applying an integral approach to statistics production, this does not mean that everything has to be designed at the same time. Rather, one can begin by putting up a framework which gives overall guidance when successively redesigning the production processes of the organisation, in such a way that the strategic goals of the organisation are met. Such a framework is called an enterprise architecture.

In the enterprise architecture of SN, the strategic goals are translated into a future model of making statistics as well as a number of principles that underpin this model. Naturally, this future model provides for the possibility of integrated production processes. It serves as a reference framework for redesigning statistical production processes and their supporting IT systems. The reference framework is mandatory in the sense that redesigned processes have to be consistent with the enterprise architecture on the basis of 'comply or explain'. A governance process has been implemented accordingly. This process is described in Section 5.

Within an enterprise architecture, a distinction can be made between an overall business and information architecture on the one hand, and a software and infrastructure architecture on the other hand (Van 't Wout et al. 2010). The software and infrastructural 
needs follow the business and information needs. In this article we focus on the business and information architecture (Huigen et al. 2006/2009). We will broadly describe the contents of the architecture as developed by SN, emphasizing its key features. The architecture will be illustrated by an example in the domain of economic statistics, showing the added value of this approach.

\subsection{Future Model of Making Statistics}

The future model of producing statistics is founded on a number of key principles. We briefly mention the four most important ones.

- Output orientation. SN wishes to publish relevant statistical information of high quality, tailored to user needs. These needs are leading when (re)designing statistical production processes within budgetary constraints. This implies that the output requirements need to be specified at the earliest stages of the redesign. The specifications include quality requirements. Legal obligations, in many cases captured in European regulations, are to be met at all times. Other less formal information needs must be established and formally decided on by $\mathrm{SN}$ on the basis of cost-benefit considerations given the strategic goals.

- Accessibility and comprehensiveness of the statistical information. For users of statistical information it is important that the information is both accessible and comprehensive. This is true for external users as well as for staff within SN that make use of statistical information as input for further processing. The principle not only implies that the populations, the variables and the reference periods to which the data relate are clearly defined, but also the quality of the data. The relevant quality dimensions depend on the use to be made of the information. Such data describing information is called metadata. When (re)designing a production process, this kind of metadata needs to be considered explicitly: there is no data without metadata. Although this may perhaps seem obvious, practice shows that it is often not so easy to comply with this principle. It also contributes to the strategic goal to improve coherence.

- Reuse of data and metadata. The policy at SN for data collection stipulates never to ask for information by questionnaire if that information is available in any administrative register. Only if it is unavoidable can information be collected directly from respondents, but even then the information can only be asked for once. Moreover, the use and reuse of source data and intermediary results by different internal users not only supports reduction of the reporting burden, but also contributes to higher cost effectiveness. A prerequisite for reusing data and metadata is that the available statistical information is accessible to internal users (see previous principle). Another prerequisite is, of course, that there are no legal obstacles to reusing data. Fortunately, the Dutch Statistics Act actually promotes the reuse, for statistical purposes, of data by $\mathrm{SN}$.

- Modular production lines. The future statistical production processes are designed by making use of customisable standard modules. The resulting production lines are cost efficient and can be adapted more easily and flexibly to changing information demand. 
The model and the principles described above hold for all future statistical production processes of SN. It covers the production of both social statistics and economic statistics. But up till now, the implementation of production processes according to the enterprise architecture has focused mainly on the first three principles, as we will see in Subsection 3.3. In Section 4, the consequences of the fourth principle are discussed.

In addition to the four principles, the enterprise architecture is based on three notions that appeared to be useful in working out the future process model and underlying principles. These notions are steady state, fixed data interface and chain management. They have to do with the network aspects of making statistics, mentioned in Section 2. Statistical production processes are connected to each other when the output of one process is (re)used as input for another. For instance, data are collected only once and then reused where needed. But also processed data can be reused, and even disseminated data are used in a feedback loop when producing data on subsequent periods, or can be further processed in the context of other statistics. In this sense, the set of production processes can be seen as constituting a network. An example of such a network is given in Subsection 3.3.

In order for the output of one production process to be usable as input for another, this output has to be not only well defined, but also stable. Statistical data sets that fulfil these requirements are called steady states. They are well defined, that is, the populations, the variables and the reference periods to which the data relate are clear as well as the quality of the data. Thus, a steady state consists of statistical data and the corresponding metadata. The stability concerns their quality. In the current production lines, the quality of the data is often continuously improved, without clear standards. To the (internal) users there is much uncertainty about the version to be used as input for further processing, since there is always a new 'latest' version. In the future production lines this uncertainty is removed. Although a steady state may consist of different quality versions (for example relating to preliminary and final figures), each quality version is stable and designed in advance. For short, we will call each quality version a steady state.

For reuse it is important that the steady states are internally accessible. To that purpose the enterprise architecture of $\mathrm{SN}$ recognises a number of fixed data interfaces. Besides the (internal) archive function, a data interface facilitates the exchange of steady states between different production lines, including the metadata concerned. A data interface has two sides: one side to deliver the statistical data set and one side to retrieve it. The supplier of the data (supplying production line) delivers and the user of the data (demanding production line) retrieves. For the physical realisation of the data interfaces, an organisational unit called the Data Service Centre (DSC) has been created. It archives the steady states and facilitates the reuse of data and metadata.

The third notion is chain management. It concerns the planning and coordination of the exchange of steady states between the statistical production lines (Van Delden et al. 2009). This is distinguished from process management, which is the management within a single statistical production line with steady states as outcomes. Chain management becomes more important as the reuse of steady states grows and hence the interdependency between the statistical production lines increases, that is, when we move further away from autonomous production lines towards an integrated network. 
Figure 1 gives a schematic representation of the ideal to-be situation for the production of statistics at SN. In the figure a distinction is made between the design of the production processes (upper layer), the management within and between the production processes (middle layer) and the implementation of the production processes (lower layer). The diamond shapes represent activities, while the rectangular shapes represent information products.

On the right-hand side, the design of the production processes starts by exploring the information needs. Based on these information needs the steady states including quality standards, as well as the production processes and methodology are designed, depicted as 'design data' and 'design process', respectively. Thus, the design is output oriented in accordance with the first principle, and is restrained by the strategic aims of the organisation and by policy, in particular budgetary resources. The design of the data results in the specifications of the steady states. The design of the statistical production process results in production rules for the modules mentioned in connection with the fourth principle above. This is elaborated in Section 4. The specifications of the steady states and the production rules are both called metadata.

The implementation of the statistical production process actually produces the steady states and consists of working through a series of activities from data collection through dissemination. Note that the figure does not depict the dynamic nature of the statistical production process as it is possible to go repeatedly through parts of the process by reusing data.

Data to be shared among different production lines are exchanged through fixed data interfaces, symbolized at the bottom of the figure by 'data (steady states)'.

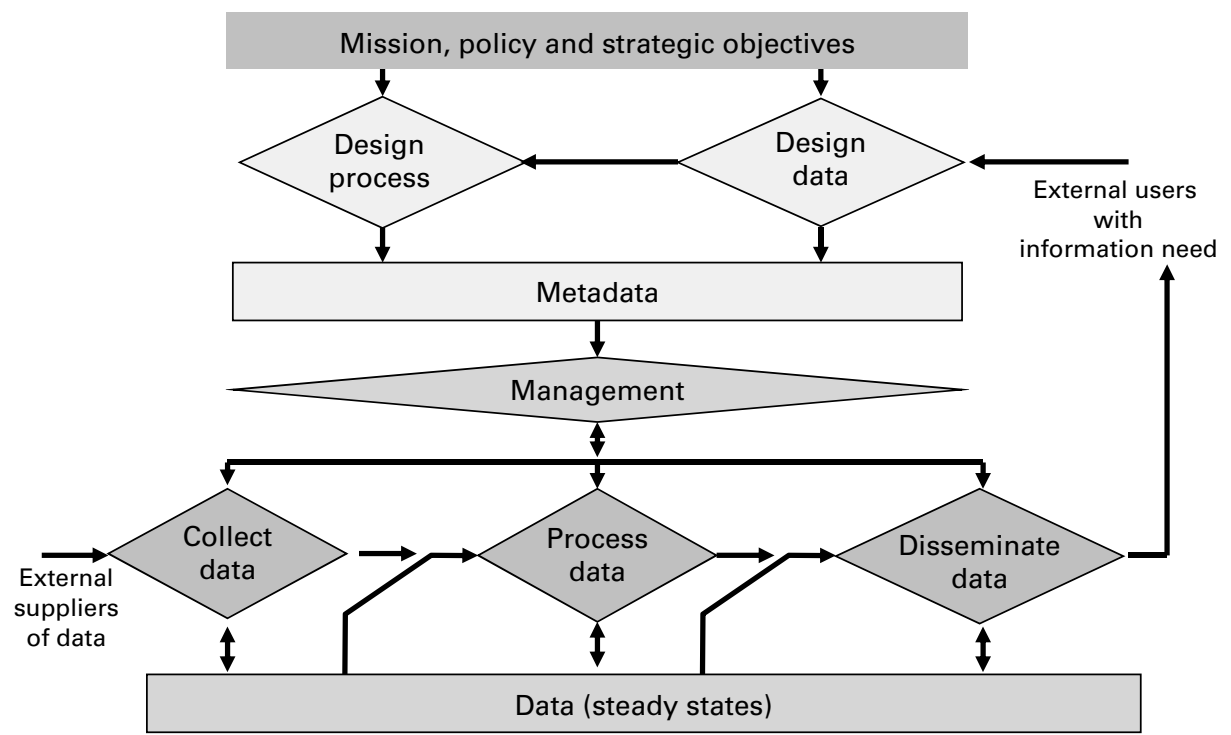

Fig. 1. (Simplified) model of future production of statistics at SN 


\subsection{Applying the Enterprise Architecture in Projects}

At $\mathrm{SN}$, redesign of statistical production processes always takes place in the form of projects. Related redesign projects are brought together under a programme in order to coordinate the dependencies between the projects and the deployment of resources. Usually the following disciplines are involved in a redesign project:

- Business analysts and project architects, who design the statistical production processes, compliant with the business and information architecture.

- Methodologists, who are responsible for sound methodology.

- Information analysts and software architects, who define the (IT-)use cases, the logical view and the implementation view of the production line to be built, compliant with the software and infrastructure architecture.

- Software developers and testers, who actually build and test the production lines.

- Statisticians, who are eventually going to use the production lines and who feed the project with subject-matter knowledge.

When applying the enterprise architecture in actual redesign projects (for an example see the next subsection), we encountered a number of practical issues. We mention the most important ones. First, the architecture reflects the corporate interest of SN. Often no local need to apply the architecture is perceived. Second, the architecture was initially developed and formulated top-down, without much explanation as to how and why it should be applied in practical situations. This led to misunderstandings. Third, there is a need for feedback to those who design the architecture from the level of application - by various disciplines that are involved in the redesigns projects - in order to get a practical set of architectural guidelines. These issues are related and show that the top-down approach has to be balanced with a bottom-up approach.

In order to overcome these issues, a number of measures were taken. One was to form multidisciplinary project teams consisting of all the disciplines described above, including explicit involvement of project architects. A second measure concerned the governance structures that were set up. This is the subject of Section 5, which describes the context in which redesign projects operate. Feedback information from both operational and strategic areas has resulted in a number of improvements in the architecture.

\subsection{Applying the Enterprise Architecture: an Example}

The enterprise architecture has been applied and further elaborated in a programme for the redesign of economic statistics (Braaksma 2007; Braaksma and Buiten 2012). This programme ran from 2007 to 2011. It focused on the core of statistics relating to economic growth, in particular annual business statistics and related short-term turnover statistics, which are covered by two European regulations: the Structural Business Statistics (SBS) and the Short Term Statistics (STS) regulation respectively. The system of National Accounts also fell within its scope. The programme centred on three strategic goals: increase efficiency ${ }^{2}$, reduce administrative burden and improve quality.

\footnotetext{
${ }^{2}$ That is, to reduce the related staff by some $20 \%$, from $325 \mathrm{fte}$ to $260 \mathrm{fte}$.
} 


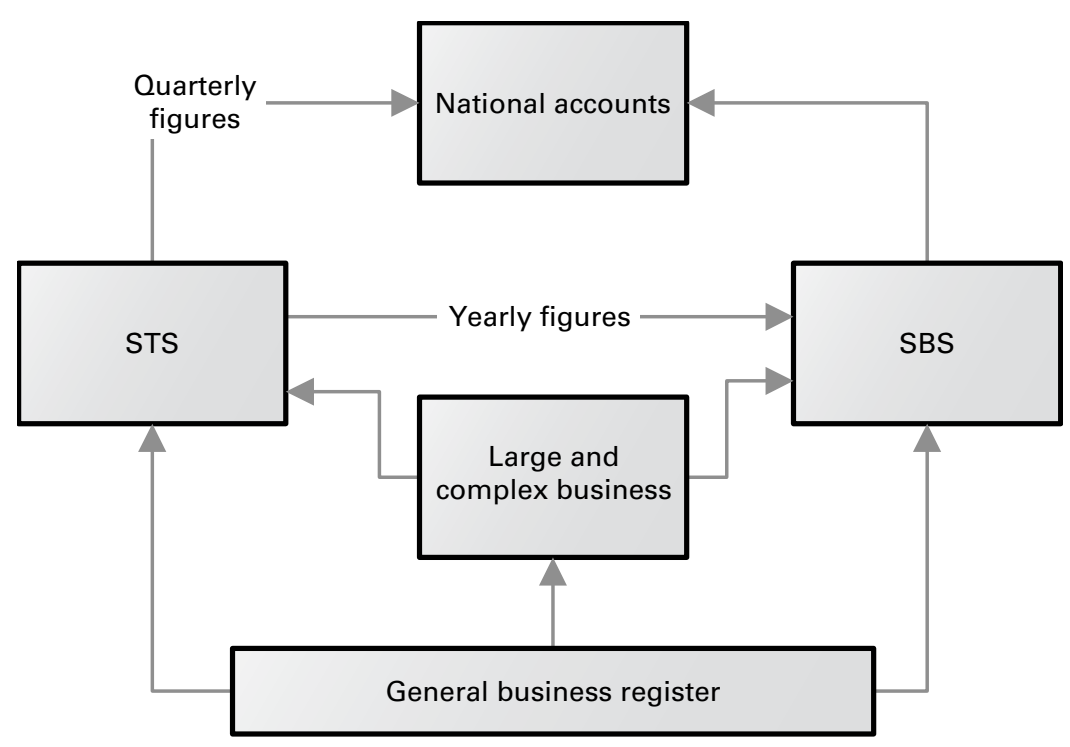

Fig. 2. Dependencies within a set of production lines for economic statistics

This redesign programme explicitly followed an integrated approach based on a comprehensive architecture for the statistics concerned. That is, the compilation of the different economic statistics is no longer designed as a set of separate production lines (stovepipes) but rather as an interconnected set of related statistical production processes. Figure 2 shows the most important statistical production processes and their interdependencies. In the following we concentrate on the STS part of the programme.

In the context of the STS regulation, SN publishes the period-to-period growth rates of monthly, quarterly and yearly turnover for four major economic domains: manufacturing industry, retail trade, construction and other services (e.g., transport). Turnover corresponds to market sales in goods and services to third parties. These high-profile short-term statistics are used to analyse and monitor current economic developments at both the national and European level.

The STS output data are growth rate indices, which provide information as to how turnover has risen or fallen compared to the previous period (month or quarter). Information on the structure of the economy for a specific year is provided by the annual production statistics based on the SBS regulation. The STS and SBS, in turn, form input for the National Accounts. For example, the short-term turnover statistics are used as a source for the compilation of quarterly Gross Domestic Product (GDP) estimates.

Before the redesign, there were coherence problems between the turnover growth figures produced by STS and those of SBS and National Accounts. The differences were especially marked for large and complex business units. The coherence problems were difficult to trace and solve, because the STS and SBS production lines were designed independently of each other. In the new design, data for the large and complex business units are collected and processed in a separate production line, generating turnover figures which STS and SBS are obliged to use. This partly solved the coherence problems. To further deal with the inconsistencies, the STS turnover figures for the smaller and less 
complex business units are used by SBS to calibrate its yearly figures. Furthermore, the STS figures are now mainly based on value added tax (VAT) data instead of survey data. Use of the VAT register also added to the reduction of the reporting burden, another important aim of the redesign.

Central to this way of compiling economic statistics is the General Business Register, maintained by SN. From this register, population frames are derived once a month and these are used to delineate the populations of STS, SBS and the large and complex businesses in a coordinated way. They also provide a central linkage of business units to administrative units, that is, fiscal units in the VAT register.

The interrelated production lines described above and depicted in Figure 2 were designed simultaneously in two stages. In the first stage, in line with the architectural principles, the separate products to be generated by the production lines for STS, SBS, large and complex businesses and the General Business Register were specified in detail, including quality requirements and delivery dates. This resulted in properly defined steady states relating to the different periodic growth figures and the population frames that could be exchanged according to the agreed delivery dates. In fact, different quality versions of the steady states were defined. All parties involved, including National Accounts as a major user of the turnover figures, collaborated in this stage, which can be seen as a form of chain management by matching demand and supply. In addition, agreements were made on error handling in the monthly population frame and about dealing with (too) large differences between the turnover totals of STS based on VAT data and SBS based on survey data. Note that the new design has not completely eliminated the inconsistencies between STS and SBS. This first stage corresponds to the component 'design data' in Figure 1.

Given these joint agreements on the data to be produced, that is the steady states, the individual production lines could be further designed relatively independently of each other in a second stage. In Figure 1, this stage is represented as 'design process'. If data has to be exchanged between various users the production lines are ideally connected to a standard data interface. However, at the time the programme was carried out, this principle did not have the highest priority. The standard data interfaces, which are realised by the DSC (see Subsection 3.1 above), were not fully operational. Similarly, the various production lines were designed as much as possible according to the same pattern, but not modular. The approach of modular production lines is still in its infancy. In the next section we will elaborate on this approach.

\section{Modular Production Lines and Standardisation}

The architectural principle of using standard modules for statistical production lines may seem obvious, since the reuse of parts of production lines has a high potential for cost reduction. However, there are a number of complicating factors. First of all, the modules can be viewed either from a business (process) perspective or from an IT (system) perspective. These different viewpoints are not always recognised and may cause miscommunications. In both approaches it is difficult to choose the most useful modules. Especially for the IT modules, complications become apparent when considering the degree of parameterisation, performance requirements and integration problems between 
modules. Moreover, there is a limit where further standardisation is no longer efficient in terms of costs or flexibility. We consider achieving optimal standardisation a main challenge of the redesign programme of SN. We have converged to the following approach, which starts from a business perspective rather than an IT perspective.

Roughly, when designing a modular production process we find it useful to distinguish between the design of the 'know' and its 'flow'. The know involves the choice and specification of the statistical methods, such as imputation methods or estimation methods. The flow involves the deployment of the know: the process modules in which the statistical methods are encapsulated, the order of these modules, the decision points that manage the statistical data through the modules, the triggers to start or end a module, and the process indicators to manage the flow as a whole. A trigger may be quality driven, cost driven, time driven or event driven.

Note that the process modules provide the link between the know and the flow. We will explain this by means of an example. Suppose we have a statistical dataset which has to be validated and corrected for so-called 1000-errors, such as made by respondents to surveys who overlook that turnover has to be given in thousands of Euros. In order to do so, a standard statistical method is to compare the data in this dataset, that have to be validated and corrected, with reference values. The discrepancy with the reference value is compared to a norm. If the discrepancy is too high, a correction is carried out. If this is not the case, the value is retained. Quality indicators are used to indicate the validation or correction of the data. So the process module has an input value, a reference value and a discrepancy norm as inputs, and an output value and a quality indicator as outputs for each data item that has to be processed. The input values are assumed to be available. A common method to obtain reference values is to take group means. The question is whether these group means should be determined within the validation module or that we should define a separate averaging module for obtaining group means; or even more generically, a module to estimate population means/totals. This example shows the difficulty of choosing the scope of an individual module and the level of granularity of the set of modules.

Although statistical methods are a key factor when constructing modules, the architecture itself does not make methodological choices. Instead, it prescribes the standard methods that may be used. Recently, the enterprise architecture of SN has been complemented by a series of standard methods. This series can be seen as a catalogue of validated and approved statistical methods, presently used at SN, that can assist in the design of a production process. By using only approved methods included in the series, the quality of the output of a production process can be better substantiated. A first version of the series was completed in 2010, and will be updated at regular intervals with new and improved methods. For example, a method to validate and correct 1000 -errors is described in this series.

The process modules described above are the natural starting point to define IT modules. These IT modules do not necessarily correspond one-to-one to the process modules. Because of performance reasons, IT modules may consist of more than one process module. If process modules are commonly used together in statistical production processes, this may be taken into account when building IT modules. 
An important consequence of the modular approach is that IT modules should be rule based. Standard methods, and the process modules that use them, can involve complex parameterisation (or specification). We call an IT module rule based if its control parameters can be specified by non-IT specialists (for example subject-matter specialists or methodologists) by means of an easily accessible interface, without need to change the internal code of the IT module itself. The extent to which an IT module should be rule based is an implementation choice, and touches on the issue of local and global interests. This choice depends on implementation costs, maintenance costs and the expected need to adjust the specifications. In general, the implementation costs will rise as the IT module is more rule based. Similarly, the costs for specification of rules will rise. But on the other hand, the more generic rule-based IT modules can be used in more different statistical production lines.

A key issue to consider is interoperability. When putting together different IT modules into a single statistical production line, one might encounter a problem of integration because the IT modules speak different languages and do not fit seamlessly together. This requires either a conversion effort between IT modules or a standardised exchange format ('plug and play').

In order to facilitate the (re)design of statistical production processes, one could consider developing a library of standard process modules. A number of potential candidates is currently being identified and described (Camstra and Renssen 2011). In addition to this library of standard process modules, one may also consider a library of standard IT modules. These IT modules are linked to the process modules, are rule based and are interoperable. The IT modules can be exploited to quickly and efficiently design and build a rule-based modular production line. By adjusting the rulebased specifications, it would be possible to quickly and flexibly respond to changing information demand.

To give an example, SN has recently developed an IT module for macro editing (Ossen et al. 2011). The main feature of this module is to validate statistical data an aggregate level and if desirable to zoom in and edit the data at the micro level. This module is flexible in the sense that users can specify their own levels of aggregation for different types of units. As a policy, the degree of flexibility of an IT module should be such that it is applicable in at least two statistical production processes. This is also the minimum rate for developing a standard IT module. The idea is that other statistical production processes will follow. Naturally, this is stimulated during the redesign projects. Currently, one year after the release of the IT module for output validation, it has been successfully integrated in four statistical production processes including the redesign of economic statistics described above.

We conclude that we have not solved the standardisation challenge yet, but we have made progress. Statistical methods are standardised and reused. A library of standard process modules is under development. But we do not yet have a library of standard IT modules, nor do we have rules to balance costs, flexibility to accommodate changes in available input or required output, and other aspects that are relevant when pursuing standardisation. This means that in practice judgmental choices have to be made when redesigning statistical production processes. For this, SN has put a governance structure into place. This is the subject of the next section. 


\section{Governance and Organisation}

When embarking on a redesign of multiple statistical production processes, several aspects are relevant from a managerial perspective:

- managing the portfolio of redesign projects.

- managing individual redesign projects.

- managing the enterprise architecture.

This implies a need for specific governance provisions. These provisions have to ensure that the projects are correctly prioritised and that they are efficiently executed in compliance with architectural requirements, which in turn have to reflect the strategic goals. They have to be tuned to the existing organisation, within budgetary constraints and taking into account the organisational culture.

In order to manage the portfolio of redesign projects and to manage the enterprise architecture as such, two central boards have been created, the Central Portfolio Board (in Dutch: CPR) and the Central Architecture Board (CAB), respectively. In the following subsections, the governing and organisational arrangements are described, including these two boards.

\subsection{Portfolio Management}

For the prioritisation of projects at the level of $\mathrm{SN}$ as a whole, the CPR has been established. It is chaired by the Chief Information Officer (CIO) of SN; all internal stakeholders are represented at strategic management level.

The CPR decides on the actual redesign programme at corporate level, by selecting which projects are to be executed from the proposals submitted by the various business units (who also have their own portfolio boards). Portfolio decisions are taken in the light of strategic goals, and on the basis of business cases that are provided with each redesign proposal. A business case is a comprehensive assessment of all current and future costs and benefits; this is further explained in the next section.

The CPR also allocates resources, in particular concerning IT services, statistical methodology, process development, and project management. The CPR does not actively pursue a multi-annual plan of controlled redesign of all statistical processes. Rather, business units provide requests that have been prioritised by their own portfolio boards.

\subsection{Managing Individual Redesign Projects}

Once the projects have been prioritised, they are executed following the well-known Prince 2 project management method (Office of Government Commerce 2009). This method ensures that stakeholders' interests are systematically taken into account. For each project a steering committee is established, in which the following main roles are distinguished (note that a steering committee member may have several roles at once):

- Executive

- User

- Supplier. 
The executive is usually the owner of the statistical production process to be redesigned. The executive chairs the steering committee and is responsible for the realisation of the project. The users have an interest in the deliverables of the project. For instance, they may be involved in carrying out the statistical production process concerned or make use of its output. The suppliers provide the resources necessary for the redesign project as described in Subsection 3.2. The Prince2 project management method provides for a solution mechanism for situations in which stakeholders disagree.

The use of Prince 2 in itself does not guarantee that the results of the projects are in line with the strategic goals of $\mathrm{SN}$ as incorporated in the enterprise architecture. In fact, as is the case with any enterprise architecture, compliance issues have to be dealt with (Foorthuis 2012). Therefore, in addition to the use of the Prince 2 method, there are a number of compliance requirements to which projects have to conform. These requirements stem from the architectural framework as described in Subsection 3.1 and take the shape of templates (BAD, MAD, SAD, refering to business, methodology and software, respectively; Hofman 2011) describing the main aspects of the redesigned statistical production process. These aspects are the output data of the production process, the data to be used as input, the methodology used, the process flow and the IT landscape. In effect, the templates provide guidance for applying the enterprise architecture in projects.

Once the redesigned production process is described according to the templates, it is reviewed. For this, a review procedure has been implemented (Foorthuis et al. 2009).

\subsection{Managing Architectural Issues}

Evidently, the enterprise architecture as such has to be managed. That is, formal decisions have to be taken on its contents and scope. Of course, managing the enterprise architecture also implies change management.

As mentioned in Section 3, in the enterprise architecture the strategic goals are translated into a future model of producing statistics, as well as a number of principles that underpin this model. Decision making on the architecture thus involves making explicit choices on how to interpret the strategic goals and make them operational. This includes making choices on what aspects of producing statistics to incorporate in the architecture. For instance, are the methods series or the set of standard IT tools to be included in the architecture? It also includes making choices regarding the balance between local and corporate interests. This is particularly important, since the architecture is a reference framework for the individual redesign projects. Stakeholders in the strategic goals of SN are not systematically represented in the steering committees of these projects, but their interests are served by the templates (BAD, MAD, SAD) to be used by each project.

It is important to emphasise that the enterprise architecture is a point on the horizon. It is a blueprint of the desired future situation regarding the organisation's processes and IT. As a consequence, decisions have to be taken concerning the trajectory to be followed in order to approach that point on the horizon. This gives rise to issues such as the question of what architectural principles deserve priority and the extent to which compliance with the architecture has to be enforced, in particular if services such as the DSC are not fully operational yet and the set of standard IT tools and modules is still 
being developed. In fact, the management of such 'libraries of standards' is itself an issue. And again, the choices to be made have to take into account both legitimate local and corporate interests.

The Central Architecture Board (CAB) has been created to address all architectural policy questions mentioned above. It is the board where architectural principles are prioritised and compliance issues discussed. The templates used in the redesign projects are formally approved by the CAB. It also advises on the set of standard IT tools, mentioned in Section 4. At SN, decisions on IT issues are not taken by a corporate IT board, as is done in some other countries; $\mathrm{SN}$ does not have such a board. The reason for this is that IT is seen as the solution to business issues. These have to be solved in accordance with the enterprise architecture, of which the IT architecture is a part. This makes the $\mathrm{CAB}$ an appropriate board to discuss strategic IT issues (Hofman and Van der Loo 2011).

The CAB is an advisory body of the Board of Directors and is chaired by the head of the $\mathrm{CIO}$ office of SN. All stakeholders, either with local or corporate interests, are represented at tactical management level. In fact, the $\mathrm{CAB}$ is one of the places where the tension between local and corporate interests can be addressed: those carrying the costs of complying with the architecture in individual redesign projects meet with those in charge of the realisation of strategic objectives, such as the reduction of the overall administrative burden to business or cost reduction through software standardisation. The CAB has proved to be instrumental in obtaining support for the approach to redesigning statistical production processes under architecture.

The role of cost-benefit considerations in the decision making of the CAB is discussed in the next section.

\section{Cost-Benefit Considerations}

As explained in the previous sections, the four strategic goals of $\mathrm{SN}$ are advanced by applying an enterprise architecture, promoting standardisation and implementing organisational provisions for managing redesign projects. However, the goal of cost efficiency is only reached if cost-benefit considerations are correctly taken into account when taking decisions, of course. We look at cost-benefit considerations first for the architecture, then for deciding on standardisation, and finally for project decisions on individual (re)design projects in the context of portfolio management.

The decision to start redesigning statistical production processes based on an enterprise architecture was strategic. Preparations were started in cooperation with an external partner, resulting in a so-called ICT Masterplan (Deloitte and Statistics Netherlands 2005; Ypma and Zeelenberg 2007), which envisaged an architecture for SN as a whole, the redesign of virtually all statistics under this architecture and central provisions for its implementation. The plan was not based on a detailed, quantitative, enumeration of costs and benefits through time, allowing an analysis of return on investment. Rather, preliminary studies by the external partner showed the strategic necessity to embark on the redesign of statistical production processes within an architectural framework. Basically, these studies provided the reasons for applying an integral approach, as described in Section 2. One of the supporting observations made was that 
most large information processing organisations have had similar experiences to those of $\mathrm{SN}$ : a proliferation of software tools at the end of last century, followed by a drive for standardisation.

The fact that the decision to develop and apply an enterprise architecture was based on strategic considerations and perceived logic rather than a detailed insight into costs and benefits does not mean that subsequent investment decisions cannot be based on costbenefit analyses. On the contrary, where possible, this should be done. Once the architecture is in place, two types of investment decisions can be distinguished: the decision to develop a standard process or IT module or tool, for instance for data editing, and the decision to redesign a specific statistical production process or set of processes within a common architectural framework. These two types of decision are intertwined. The benefits of a standard IT tool or module will increase with each process that is redesigned to use it, and a redesign becomes more attractive the more standard IT tools or modules are available to support it. On the other hand, investing in IT standards has to be based on the assumption that sufficiently many redesigned processes are going to use them, and investment decisions on new statistical processes must be made under the assumption of availability of necessary IT tools and modules.

\subsection{Architecture Costs and Benefits}

As already said, the decision to start developing an enterprise architecture was not based on explicit and quantitative cost-benefit considerations. It is possible, however, to give some indications on both aspects in hindsight. Below we give an overview of costs that have been made and benefits that have been realised so far.

On the cost side, we can distinguish between initial investment costs, maintenance costs and support costs. The initial investment concerned a core team of two external consultants and three internal experts who worked almost full time during a six-month period in 2006 on drafting the enterprise architecture. For the business architecture in particular a lot of pioneering work had to be done, since no examples existed the time. Many elements had to be developed from scratch. Many other people were involved part time, for example for reviewing drafts and for supplying subject-matter background knowledge.

After the initial development phase, a small team of architects was established including two lead architects (one for the business architecture, one for the IT architecture). Their task is to maintain, promote and further develop the enterprise architecture. They also prepare the meetings of the $\mathrm{CAB}$. The architects combine their architectural work with other duties like consultancy, research, business analysis and software development.

Another cost element is training in architecture. A first batch of people was trained by external consultants using a standard off-the-shelf course. This was considered not sufficiently tailored to internal needs and therefore in 2009 a three-day course on business architecture was developed in-house. The internal course has since been given annually for staff who need more than a casual understanding of architecture (business analysts, methodologists, software developers). They highly appreciated the course.

Thus, the bulk of costs concerns human resources. Apart from this, some limited costs arise from software tools supporting architecture development. 
On the benefit side, most rewards materialise through application of the enterprise architecture in concrete (re)design projects. Various benefits are reaped when applying the architectural approach: for example, statistical production processes run more smoothly, cost less, are better integrated into the existing environment and are more transparent, which in turn enhances quality and speed and reduces risks. It is difficult, however, to quantify such benefits since most of them are not easily measured, the role of architecture is intertwined with other aspects (like project management methods, software development methods) and we cannot conduct falsifiable or repeatable experiments. This situation is acknowledged in the scientific community (Liimatainen 2008), although some attempts are being made to measure benefits (Boucharas et al. 2010).

Larger-scale redesign programmes may reap additional benefits from an architectural approach by developing a programme architecture at an early stage. A clear example is the redesign of economic statistics introduced in Subsection 3.3, which has benefited greatly from the comprehensive programme architecture that was developed in early 2007 . The programme architecture enabled integral management of the development programme, and scoping of individual projects was simplified. Moreover, a new and efficient organisational structure for economic statistics has been created along the lines of the programme architecture. And while the main role of the programme architecture was at design time, it still plays a part at run time. This is most obvious in chain management, where the concept of steady state is rather important and is used heavily to manage quality expectations and realisations.

Furthermore, an important indicator for the perceived benefits of the enterprise architecture is its acceptance by the business, in particular key players in subject-matter domains. An audit by a team containing external auditors showed general appreciation among persons interviewed (internal report by the audit team, consisting of D. Baart, A. Cholewinska, J. Jansen and Y. Vergeer, 2011). In particular, the steady state concept was highly valued. Concerns expressed relate for example to scalability - how much architecture is needed for smaller projects? - and communication. The fact that appreciation for the architecture has increased is probably due to a number of key factors. First, the business now better understands the enterprise architecture and its uses. Second, a continuous dialogue and interaction of architects with end users has helped to improve the architecture and the way it is being applied. Third, the continued support and commitment from strategic management for the architectural approach, as seen in the $\mathrm{CAB}$, was crucial.

When the notion of architecture was first introduced, it met with scepticism and its added value was questioned. The scepticism has largely disappeared over the years and the added value is now broadly recognised. Architecture has become an established discipline.

\subsection{Standard IT Tools and Modules}

As to standard IT tools and modules, it has proved difficult to give decision making a sound financial footing, although development or purchase costs can be estimated. A wellfounded judgment requires an overview of processes where a potential standard tool or module is to be applied, insight in the costs of implementation (including interfaces), an idea of training costs, a comparison of old versus new maintenance costs, life cycle 
estimates, and so on. As a result, decision making on IT tooling is based on qualitative rather than quantitative considerations.

Applying potential new standard IT tools or modules in a pilot setting may help to get a feeling of the costs and benefits involved. This approach is implemented as one of the key elements of the Innovation Programme that Statistics Netherlands has started in 2012 (Braaksma et al. 2012). Although the experience at the time of writing is still limited, already a number of successful pilot studies of potential standard IT tools and modules have been carried out, in close cooperation between potential users and IT staff. The fact that potential users are actively involved helps to ensure that a new tool actually satisfies business needs and hence improves the chances that it will be actually used. One new tool (Microsoft PowerPivot, an Excel add-on for processing large datasets) is, after a successful pilot study, submitted for inclusion in the set of standard IT tools mentioned in Section 4. Others are expected to follow.

\subsection{Individual Redesign Projects}

As mentioned in the previous section, project decisions in the context of portfolio management are based on their business case. This comprehensive assessment of all current and future costs and benefits includes financial as well as nonfinancial aspects. It includes, for instance, direct project costs, current and future maintenance costs, training costs, depreciations, required staff, effect on quality, response burden, continuity considerations and strategic consequences. Proposals for redesign are usually compared to alternative scenarios, including as a minimum the scenario of not starting any change at all. A manual for writing good business cases has been developed with the help of external experts. Business cases are assessed by controllers and other internal staff. When choosing between proposals that have a valid business case, the CPR is led by strategic considerations.

In practice, the problem of interdependent business cases between projects can be dealt with by starting with an integrated business case in which costs and benefits may not be fully quantified, but in which strategic considerations are taken into account. Individual projects may then refer to the overall business case and may assume the realisation of the other projects that are covered by the overall business case. Such constrained business cases allow for effective project assessments at their inception as well as during their execution. Moreover, the overall business case can be updated at intervals. When redesigning the chain of economic statistics, as discussed in Subsection 3.3, this was the approach taken.

As to quality, it was assumed that redesigned statistics would at least retain their previous level, in accordance with the second strategic goal as mentioned in Section 1. This assumption was not further specified, although the quality aspect of coherence was expected to improve. Actually, apart from sampling errors and response data, for many statistics most other quality dimensions are not routinely quantified. It was envisaged that applying architectural standards, such as specifying the quality dimension of datasets, would help reduce the weaknesses concerning quality information of statistics (Struijs 2005). For example, in the redesign programme of the chain of economic statistics this was taken into account by explicitly addressing quality indicators in chain management and its 
procedures. Unfortunately, it is too early to draw conclusions about the actual effects of the redesign of statistical production processes on quality, because results are only now emerging. The proof of the pudding will be in the eating: it is up to the users of the statistical information to assess the results of the redesign efforts.

\section{Experiences and Lessons Learned}

Redesigning statistical production processes within a common enterprise architecture has been underway for about seven years. What can be learned from the Dutch experiences? On the positive side, we conclude that an enterprise architecture for an NSI can be developed and applied. Although not fully mature, the architecture of SN is accepted throughout the institute as the mandatory reference for the redesign of statistics.

What has actually been achieved? SN has set up a Central Architecture Board (CAB) to address all architectural policy questions. Templates are available to guide the redesign projects. These templates are approved by the $\mathrm{CAB}$ and incorporate the essence of the enterprise architecture. For example, the principles of output orientation and comprehensiveness of statistical information are embodied in the templates. The notion of steady state has proved to be most useful in redesign projects, as illustrated by the example in Subsection 3.3. It enforces discipline: those responsible for statistical production processes have to explicitly specify their products and their potential customers. SN has implemented a Data Service Centre (DSC) to archive and exchange steady states, although its actual use has so far been limited to the archive function.

Of course the enterprise architecture is not an end in itself, but should be instrumental in bringing the strategic aims of the organisation closer to realisation. The question is to what extent the enterprise architecture has been successful in doing this. Due to the application of the concept of steady states, the comprehensibility of the data has been improved as well as the transparency of the production process. This has primarily advanced the goals of high quality standards and cost efficiency.

What has been achieved so far can be seen as an important first stage. We feel much more can be achieved to advance the strategic goals, in particular the cost efficiency. After all, the enterprise architecture only outlines a point on the horizon. In order to get closer to this future situation we need to further develop the DSC so as to facilitate the exchange of steady states. Furthermore, we have to invest in the libraries of process and IT modules, but there are still many issues in this area to be addressed. On the one hand, these issues concern the difficulties in identifying reusable modules and choosing the optimal degree of (rule-based) parameterisation. On the other hand, for many modules the business case is not well established. Especially for the IT modules the investment costs are considerable. In this area, there may be opportunities for international cooperation. This will alter the business case, since investment costs will be shared. But this requires considerable coordination effort.

Many institutes face the same challenges. In fact, they have started cooperating internationally. See for example the vision of the High-Level Group for strategic developments in Business Architecture in Statistics, established in 2010 by UN/ECE (HLG-BAS 2011). As a start, this vision resulted in a common language and structure regarding statistical process and information modelling. The international reference for 
process modelling is GSBPM and for information modelling the newly developed GSIM (Generic Statistical Information Model). A joint study conducted by Scandinavian countries and the Netherlands (Booleman and Linnerud 2010) has shown that the NSIs of these countries already have similar architectures when mapped onto GSBPM, which could be a good starting point for identifying promising cooperation efforts.

The achievements described above have not been realised in a straightforward way. We have learned a number of lessons, some positive, some negative, that deserve to be made explicit for the benefit of other NSIs. We mention the following three:

- Initially, support for an enterprise architecture was not general. Backing by the top level appeared to be essential to get over the critical point. The CAB played a crucial role in adapting and accepting the enterprise architecture. Two shortcomings in the architecture had to be corrected. First, the enterprise architecture focused too much on corporate interests and neglected the local interests. Second, the enterprise architecture was developed and formulated top-down, without much practical explanation as to how and why it should be applied.

- Complying with the enterprise architecture requires the fulfilment of certain organisational arrangements. For instance, compulsory use of standards implies the availability and accessibility of these standards. The same holds for reuse of data. As long as these organisational arrangements, like the DSC, are not fully implemented, there is much miscommunication and frustration when these standards are prescribed. In fact, the lack of the necessary organisational arrangements affects the business case of an enterprise architecture negatively.

- Although a good business case is essential for a good project, there are two caveats when applying it in the context of the redesign portfolio. First, not all costs and benefits can be expressed in financial terms, forcing a comparison of apples and oranges and causing a bias in favour of measurable aspects. Second, costs and benefits may depend on the realisation of other projects and vice versa. In such cases an integrated business case is called for, which may be awkward from a managerial point of view. In particular, standardisation benefits materialise only if adoption is sufficiently massive. This does not mean that the effort of specifying all costs and benefits does not make sense. It does, but the available information has to be used wisely.

How to proceed? Directed by the $\mathrm{CAB}$ and in cooperation with the business units concerned we would opt for an incremental approach. For example, we could first further develop reusable process modules and apply them in actual redesigns. Only after successful evaluation is it worthwhile to move to the next level and focus on creating reusable IT modules, starting with a proof of concept. Implementation of an IT module as a standard depends on cost-benefit considerations.

\section{References}

Bae, L.-C. (2010). Building an Enterprise Architecture of Statistics Korea. Paper presented at the Meeting on the Management of Statistical Information Systems (MSIS 2010), Daejeon, 2010. Available at: http://www.unece.org/fileadmin/DAM/stats/documents/ ece/ces/ge.50/2010/wp.7.e.pdf (accessed January 29, 2013). 
Booleman, M. and Linnerud, J. (2010). Cooperation Based on the GSBPM. Paper presented at the Work Session on Statistical Metadata (METIS), Geneva. Available at: http://www.unece.org/fileadmin/DAM/stats/documents/ece/ces/ge.40/2010/wp.12.e.pdf (accessed January 29, 2013).

Boucharas, V., Van Steenbergen, M., Jansen, S., and Brinkkemper, S. (2010). The Contribution of Enterprise Architecture to the Achievement of Organizational Goals: Establishing the Enterprise Architecture Benefits Framework. Technical Report UU-CS-2010-014 June 2010, Department of Information and Computing Sciences, Utrecht University. Available at: http://www.cs.uu.nl/research/techreps/repo/CS-2010/ 2010-014.pdf (accessed January 29, 2013).

Braaksma, B. (2007). Redesign of the Chain of Economic Statistics in the Netherlands. Paper presented at the Seminar on Registers in Statistics, Helsinki. Available at: http:// www.stat.fi/registerseminar/sessio3_braaksma.pdf (accessed January 29, 2013).

Braaksma, B. (2009). Redesigning a Statistical Institute: The Dutch Case. In: Proceedings of MSP2009, Workshop on Modernisation of Statistics Production 2009. Available at: http://www.pubkat.scb.se/Grupp/Produkter_Tjanster/Kurser/ModernisationWorkshop/ final_papers/H_2_business_models_Braaksma_final.pdf (accessed January 29, 2013).

Braaksma, B. and Buiten, G. (2012). Redesign of the Chain of Economic Statistics in the Netherlands. Paper presented at the Fourth International Conference on Establishment Surveys, Montreal.

Braaksma, B., Colasanti, C., Falorsi, P.D., Kloek, W., Vidal, M.M., Museux, J-M., and Szep, K. (2013). Standardisation in the European Statistical System. Paper prepared for the NTTS 2013 conference. Available at: http://www.cros-portal.eu/sites/default/ files/NTTS2013fullPaper_202.pdf (accessed March 1, 2013).

Braaksma, B., Verbruggen, M.H.M., Heerschap, N.M., and Roos, M.R. (2012). Innovation at Statistics Netherlands. Paper presented at the UN/ECE Seminar on New Frontiers for Statistical Data Collection, Geneva. Available at: http://www.unece.org/fileadmin/ DAM/stats/documents/ece/ces/ge.44/2012/mtg2/WP7.pdf (accessed January 29, 2013).

Camstra, A. and Renssen, R. (2011). Standard Process Steps Based on Standard Methods as Part of the Business Architecture. Paper presented at the 2011 ISI Conference, Dublin. Available at: http://isi2011.congressplanner.eu/pdfs/650238.pdf (accessed January 29, 2013).

Deloitte and Statistics Netherlands (2005). Excerpt from the ICT Masterplan "Counting on Statistics". Report prepared for the Advisory Council on Methodology and Information Technology.

Doherty, K. (2010). How Business Architecture Renewal is Changing IT at Statistics Canada. Paper presented at the Meeting on the Management of Statistical Information Systems (MSIS 2010), Daejeon, 2010. Available at: http://www.unece.org/fileadmin/ DAM/stats/documents/ece/ces/ge.50/2010/wp.2.e.pdf (accessed January 29, 2013).

Engelbertink, F.P. and Vogt, H.H. (2010). How to Save on Software Maintenance Costs. White Paper, Omnext BV, Veenendaal. Available at: http://www.omnext.net/ downloads/Whitepaper_Omnext.pdf (accessed January 29, 2013).

Falorsi, P.D., Barcaroli, G., Fasano, A., and Mignolli, N. (2013). A Business Architecture Framework for Industrialisation and Standardisation in a Modern National Statistical 
Institute. Paper prepared for the NTTS 2013 conference. Available at: http://www.crosportal. eu/sites/default/files/NTTS2013fullPaper_218.pdf (accessed March 1, 2013).

Foorthuis, R.M. (2012). Project Compliance with Enterprise Architecture. Doctoral dissertation ( $\mathrm{PhD}$ thesis), Utrecht University, Department of Information and Computing Sciences, Center for Organization and Information. Available at: http:// igitur-archive.library.uu.nl/dissertations/2012-1008-200504/foorthuis.pdf (accessed January 29, 2013).

Foorthuis, R.M., Hofman, F., Brinkkemper, S., and Bos, R. (2009). Assessing Business and IT Projects on Compliance with Enterprise Architecture. In: Proceedings of GRCIS 2009, CAiSE Workshop on Governance, Risk and Compliance of Information Systems. Available at: http://tunguska.home.xs4all.nl/Publications/Docs/Assessing\%20Business $\% 20$ and $\% 20$ IT $\% 20$ Projects\%20on\%20Compliance\%20with\%20EA\%20-\%20Foor thuis\%20et\%20al.pdf (accessed January 29, 2013).

Galvin, V. and Rhodes, N. (2010). Using the Idea of Statistical Architecture at Statistics New Zealand. Paper presented at the 12th East Asian Statistical Conference, Tokyo, 2008. Available at: http://www.stat.go.jp/english/info/meetings/eastasia/pdf/t2nzpa.pdf (accessed January 29, 2013).

HLG-BAS (2011). Strategic Vision of the High-Level Group for Strategic Developments in Business Architecture in Statistics. UN/ECE. Available at: http://www1.unece.org/ stat/platform/display/hlgbas/Strategic + vision + of + the + High-level + group + for + strategic + developments + in + business + architecture + in + statistics (accessed January 29, 2013).

HLG-BAS (2012). Strategy to Implement the Vision of the High-Level Group for Strategic Developments in Business Architecture in Statistics. UN/ECE. Available at: http://www1. unece.org/stat/platform/download/attachments/65962687/10+-+ HLGBAS+ Strategy. pdf?version=1\&modificationDate= 1336403439393 (accessed January 29, 2013).

Hofman, F. (2011). Redesign Approach at Statistics Netherlands. Paper presented at the 2011 International Methodology Symposium, Statistics Canada, Ottawa Canada.

Hofman, F. and Van der Loo, M. (2011). Standardisation and Differentation in Redesigns. Statistics Netherlands. Available at: http://www.cros-portal.eu/sites/default/files// Standardisation \%20and\%20Differentiation\%20in\%20Redesigns\%20v1.0p3.doc (accessed January 29, 2013).

Huigen, R., Bredero, R., Dekker, W., and Renssen, R. (2006/2009). Statistics Netherlands Architecture; Business and Information Model. Discussion Paper 09018, Statistics Netherlands, The Hague. Available at: http://www.cbs.n1/NR/rdonlyres/ A6F67DFB-859A-4F3E-83A0-ED8055659D4E/0/200918x10pub.pdf (accessed January 29, 2013).

Lankhorst, M. (2009). Enterprise Architecture at Work: Modelling, Communication and Analysis. Berlin: Springer Verlag.

Liimatainen, K. (2008). Evaluating Benefits of Government Enterprise Architecture. Paper presented at the 31st Information Systems Research Seminar in Scandinavia, Åre. Available at: http://www.iris31.se/papers/IRIS31-059.pdf (accessed January 29, 2013).

Lorenc, B., Fossan, Y., Holm, E., and Jansson, I. (2011). Towards an Enterprise Architecture for an NSI. Available at: http://www.cros-portal.eu/sites/default/files/ S4P2.pdf (accessed January 29, 2013). 
Office of Government Commerce (2009). Directing Successful Projects with PRINCE2 ${ }^{\mathrm{TM}}$ London, UK: The Stationery Office.

Ossen, S.J.L., Hacking, W.J.G., Meijers, R.J., and Kruiskamp, P.H. (2011). MacroView: a Generic Software Package for Developing Macro-Editing Tools. Paper presented at the Work Session on Statistical Data Editing, Ljubljana. Available at: http://www.unece.org/ fileadmin/DAM/stats/documents/ece/ces/ge.44/2011/wp.14.e.pdf (accessed January 29, 2013).

Struijs, P. (2005). Improving the Quality of Statistics through the Application of Process Methodology. Paper prepared for the Advisory Council on Methodology and Information Technology.

Studman, B. (2010). A Collaborative Development Approach to Agile Statistical Processing Architecture - Australian Bureau of Statistics (ABS) Experience and Aspirations. Paper presented at the Meeting on the Management of Statistical Information Systems (MSIS 2010), Daejeon. Available at: http://www.unece.org/ fileadmin/DAM/stats/documents/ece/ces/ge.50/2010/wp.3.e.pdf (accessed January 29, 2013).

Swiss Federal Statistical Office (2007). Conceptual Architecture of the Statistical Information System at the Swiss Federal Statistical Office. Available at: http://unstats. un.org/unsd/dnss/docViewer.aspx?docID = 1564 (accessed January 29, 2013).

Vale, S. (ed.) (2009). Generic Statistical Business Process Model, Version 4.0. Joint UNECE/Eurostat/OECD Work Session on Statistical Metadata (METIS), Eurostat, Luxembourg. Available at: http://www1.unece.org/stat/platform/download/ attachments/8683538/GSBPM+Final.pdf?version=1 (accessed January 29, 2013).

Van Delden, A., Lammertsma, A., and Van de Ven, P. (2009). Chain Management in Statistics: Best Practices. Statistics Netherlands. Available at: http://www.cbs.nl/NR/ rdonlyres/F757EED3-7B6B-4C43-B8E2-313C3E2E4B93/0/200943x10pub.pdf (accessed January 29, 2013).

Van der Veen, G. (2007). Changing Statistics Netherlands: Driving Forces for Changing Dutch Statistics. Paper presented at the Seminar on the Evolution of National Statistical Systems, New York, February 2008. Available at: http://unstats.un.org/unsd/dnss/ docviewer.aspx?docID=1572 (accessed January 29, 2013).

Van 't Wout, J., Waage, M., Hartman, H., Stahlecker, M., and Hofman, H. (2010). The Integrated Architecture Framework Explained. Berlin: Springer Verlag.

Ypma, W.F.H. and Zeelenberg C. (2007). Counting on Statistics; Statistics Netherlands' Modernization Program. Paper presented at the UnStatCom/ECE Conference of European Statisticians, Geneva. Available at: http://www.cbs.nl/NR/rdonlyres/ 61D36740-0489-4A76-B243-E86A3BEFBBD8/0/200932x10pub.pdf (accessed January 29, 2013).

Received December 2011

Revised April 2012

Accepted November 2012 